\title{
Monocularly produced compound afterimages
}

\author{
ALEEZA CERF-BEARE \\ University of Maryland Baltimore County, Baltimore, Maryland
}

\begin{abstract}
A compound afterimage is reported after sequential presentation to the same eye of two orthogonally-oriented patterns. The compound's duration is comparable to the duration of the unitary state of an afterimage following single exposure of a two-orientation pattern, and is governed by spatiotemporal luminance parameters. It is suggested that afterimage duration measurement will lead to an understanding of afterimage visibility mechanisms while also providing noninvasive means for investigating cortical orientation processes in the spatial and temporal domain.
\end{abstract}

In this note, I introduce a previously unexplored phenomenon - the compound afterimage-which was observed in the course of experiments that measured the duration of an afterimage generated by a square-wave grating exposed at different orientations. The compound afterimage was reported by the observer when two stimuli which differed in orientation were exposed sequentially to the same eye. The afterimages of the two orientations were seen as superimposed upon one another so that the observer reported seeing a grid for given durations of the response-timing period after the two-stimulus sequence that consisted of vertically and horizontally oriented gratings. Visibility of a cross in afterimage following exposure of two orthogonally oriented lines was given brief mention by Sindermann \& Lüddeke (1972) in their discussion of monocular rivalry.

Experiments in which afterimage duration is the dependent variable commonly employ stimulus exposures designed to generate afterimages lasting up to $60 \mathrm{sec}$ (Atkinson, 1972; Cerf-Beare, 1984), followed by 5-min readaptation periods between exposures. The afterimage can be revived or prolonged, however, if the eye is restimulated before the readaptation process has been allowed to run its course (MacLeod \& Hayhoe, 1974; Magnussen \& Torjussen, 1974). The pattern compound afterimages were initially observed when the second grating was exposed after delays of 3 and $4 \mathrm{~min}$, before readaptation was complete. Exposure of the second grating can thus be said to have revived the afterimage of the first grating. The formation of a compound afterimage under these circumstances indicates storage periods for pattern information which, being beyond the scope of retinal temporal interactions, implicate cortical orientation processes (see also Corwin, Volpe, \& Tyler, 1976).

Investigations of afterimage durations following single exposures of two-orientation patterns (Atkinson, 1972; Cerf-Beare, 1984) provided evidence that the states and durations of the afterimages reflect activity of, and inter-

The author's mailing address is: Department of Psychology, University of Maryland Baltimore County, Baltimore, MD 21228. Helpful comments from Marilyn E. Demorest and Terry L. Benzschawel are gratefully acknowledged. action between, cortical orientation processes. Preliminary experiments assessing compound afterimage durations following a two-stimulus sequence (delivered without delay) now indicate that processes analogous to those following the single two-orientation exposure can be demonstrated with this technique.

Afterimage duration following exposure of a single stimulus pattern consists of two states which alternate with one another nonsystematically as long as the afterimage remains visible. These are the unitary state, in which all the components of the originally exposed pattern are present in afterimage, and the fragmentary state, in which only part of the originally exposed pattern is visible (Atkinson, 1972; Swanston \& Wade, 1981; Wade, 1972). Given the appropriate instrumentation, each state is separately measurable. The sum of the two measures yields a third, total afterimage duration measure, thus providing three measures of afterimage duration within a fixed period following stimulus exposure.

Cerf-Beare (1984) showed total visibility duration to be governed by the spatiotemporal luminance distribution, not by the orientation components, of the stimulus pattern. The unitary state occupied $65 \%$, and the fragmentary state occupied $35 \%$ of the total, irrespective of orientation changes. Specific cortical orientation processes were reflected in the relative duration generated by each of the pattern's two orientations within the fragmentary state.

The results of two preliminary experiments are summarized here. Compound afterimages were generated with a space-averaged luminance of $4.13 \times 10^{3} \mathrm{~cd} / \mathrm{m}^{2}$ following the sequential exposure of two square wave $1.5 \mathrm{cpd}$ black-and-white gratings, one in vertical, the other in horizontal orientation. Apparatus and procedure were as described by Cerf-Beare (1984) except that each of the two stimuli were placed in one channel of a dual-beam Maxwellian view system, and exposures were delivered by two sequentially-programmed Uniblitz (Vincent) shutters.

In one experiment, the afterimage states were investigated with three subjects, each making 12 observations of a 4-sec stimulus sequence ( $2 \mathrm{sec} /$ orientation). That state in which the compound afterimage was visible (i.e., the afterimages of both gratings were seen together as a grid) 
appears analogous to the unitary state. The fragmentary state would be that in which the afterimage of each grating was seen alone. Of the mean total duration of $16 \mathrm{sec}$, about $60 \%$ occupied fragmentary duration. The compound afterimage (unitary duration) was visible for $40 \%$ of the total duration. Sequence order, which had been counterbalanced over trials and subjects, had no effect on either durations or proportions. These data show that the duration proportions of the two states after sequential exposure, while not identical with those of the single exposure, are also predictable.

A second experiment investigated the temporal parameter of exposure duration by comparing compound duration following a 10 -sec ( $5 \mathrm{sec} /$ orientation) with a $400-\mathrm{msec}$ (200 msec/orientation) exposure sequence. Twenty-two observers served in both conditions. The results confirmed that compound afterimage duration was sensitive to the temporal parameter. Mean duration was $21.35 \mathrm{sec}$ for the 10-sec exposure and $11.94 \mathrm{sec}$ for the 400 -msec exposure. A repeated measures one-way ANOVA showed the difference between these means to be significant $[F(1,21)=$ $25.55, \mathrm{p}<.0001]$. Investigation continues with intermediate exposure durations and different luminance levels.

The data obtained with sequential exposure, as well as the previously cited results for simultaneous exposures, indicate that afterimage duration generated with orientation patterns provides stable and predictable measures, sensitive to both spatiotemporal luminance, and to spatial orientation parameters. Such measures therefore hold the key to an eventual understanding of the mechanisms which regulate afterimage visibility and those which organize the afterimage signal into separate component patterns. Most importantly, the techniques employed in afterimage duration measurement provide a noninvasive way to investigate cortical orientation processes as these respond to spatially and temporally varied input.

\section{REFERENCES}

AtKInson, J. (1972). Visibility of an afterimage in the presence of a second afterimage. Perception \& Psychophysics, 12, 257-262.

Cerf-Beare, A. (1984). Pattern and orientation effects on afterimage duration. Perception, 13, 443-453.

CoRwin, T. R., Volpe, L. C., \& Tyler, W. C. (1976). Images and afterimages of sinusoidal gratings. Vision Research, 16, 345-349.

MACLEOD, D. I. A., \& HAYHOE, M. (1974). Rod origin of prolonged afterimages. Science, 185, 1171-1172.

MAGNUSSEN, S., \& TORJUSSEN, T. (1974). Sustained visual afterimages. Vision Research, 14, 743-744.

SindermanN, F., \& LüddeKe, H. (1972). Monocular analogues to binocular contour rivalry. Vision Research, 12, 763-772.

Swanston, M. T., \& WADE, N. J. (1981). Consistency of response during steady visual stimulation. Perception, 10, 339-349.

W AdE, N. J. (1972). Orientation effects on line afterimages. Perception \& Psychophysics, 12, 409-416.

(Manuscript received for publication February 25, 1985.) 